Mitthellungen ans dem chemischen Institut der Universität Rostock.

(Fingelaufen am 17. September 1900.)

\title{
Ueber das Phosphorsuboxyd;
}

von A. Michaelis und K. v. Arend.

[Zweite Abhandlung 1 )].

In der ersten Abhandlung über das Phosphorsuboxyd haben Pitsch und der Eine von uns gezeigt, dass diese Verbindung erhalten wird, entweder durch Aufösen von gewöhnlichem Phosphor in wässrig-alkoholischem Alkali und Fällung mit verdünnter Säure, oder durch Einwirkung von Essigsäureanbydrid auf eine Eisessiglösung von unterphosphoriger Säure. Durch eine Reibe sorgfältig ausgeführter Analysen wurde gezeigt, dass dem Phosphorsuboxyd zweifellos die Formel $\mathrm{P}_{4} \mathrm{O}$ zukommt.

Nun hatten aber Pitsch und Michaelis anfangs ${ }^{y}$ ) in einzelnen Fällen bei der Analyse des nach der zuerst angegebenen Methode gewonnenen Pbosphorsuboxyds einen etwas zu hohen Procentgehalt an Phosphor (bis in $90 \mathrm{pC}$. statt $88,57 \mathrm{pC}$.) gefunden und ebenso geben D. L. Chapman und F. A. Lidbury ${ }^{3}$ ) an, dass der von ihnen aus der rothen Lösung gefällte Körper 90,5-91,51 pC. Phosphor enthalte. Zur Aufkläruug dieser Erscheinung baben wir eine weitere Untersuchung des Phosphorsuboxydes ausgefulhrt, die sich auch auf das vermittelst unterphosphoriger Säure hergestellte Suboxyd und auf den durch Belichtung erhaltenen, sogenannten Pedler'schen Phosphor erstreckt.

1) Erste Abhandlung: Ueber die niederen Oxyde des Phosphors, siele diese Annalen $\mathbf{3 1 0}, 45$ u. ff.

2) Ber. d. deutsch. chem. Ges. 32, 338.

3) Journ. chem. Soc. 75, 973.

Annalen der Cbewie 314. Bd. 
Chapman und Lidbury sind der Ansicht, dass der Niederschlag, der aus der rothen Lösung vermittelst verdunnter Säuren erhalten wird, nichts anderes als amorpher Pbosphor sei, obgleich ihre eigenen Analysen dem durchaus widersprechen, da sie etwa $10 \mathrm{pC}$. Phosphor weniger fanden, als der reine, rothe Phosphor enthält, dagegen nur 2 pC. mehr als dem Phosphorsuboxyd zukommt. Sie stützen diese Annahme aber weiter darauf, dass der durch Belichtung gebildete rothe Phosphor auch leicht in wässrig-alkoholischem Alkali löslich sei, wovon sie sich durch directe Versuche iberzeugten. Diese Angabe erschien uns von Bedeutung. Wenn dieselbe auch keineswegs bewies, dass der als Phosphorsuboxyd betrachtete Körper rother Phosphor sei, da sich dieser ja in irgend einer Form, ebenso wie der gewöhnliche Phosphor in wässrig-alkoholischem Kali unter Oxydation lösen konnte, so war durch diese Löslichkeit doch ein wichtiges Unterscheidungsmerkmal zwischen Phosphorsuboxyd und rothem Phosphor aufgehoben.

Bei unseren Untersuchungen in dieser Richtung ergab sich nun eine unerwartete Schwierigkeit: es war uns bis jetzt unmöglich, auch nur annähernd analysenreinen rothen Phosphor durch Belichtung zu erbalten. Der rothe Phosphor ist in dieser Weise in grösserer Menge zuerst von A. P edler durch die intensive Wirkung der Sonnenstrahlen in Indien dargestellt, leider aber nicht analysirt worden. Wir erhielten aus einer concentrirten Lösung von Phosphor in Schwefelkohlenstoff bei unseren Sonnenverhältnissen niemals reinen rothen Phosphor. War die Lösung völlig trocken, so enthielt das Präparat erhebliche Mengen von Schwefel und Kohlenstoff, war dagegen auch nur eine Spur von Feuchtigkeit vorhanden, auch Sauerstoff. Setzt man Phosphor unter Wasser der Wirkung des Lichtes aus, so entsteht fast reines Phosphorsuboxyd. Aus einer Lösung von Phosphor in Tetrachlorkohlenstoff erbält man einen kohlenstoffhaltigen Körper. Wir haben daher bis jetzt nicht feststellen können, ob reiner rother Phosphor, der vermittelst des Lichtes erhalten wurde, sich in wässrig-alkoho- 
Michaelis und v. Arend, Ueber das Phosphorsuboxyd. 261

lischem Alkali löst. Die unter Ausschluss von jeder Spur Feuchtigkeit erhaltenen Präparate waren in der alkalischen Flüssigkeit entweder nur spurenweise oder gar nicht löslich und verhielten sich jedenfalls ganz anders wie Phosphorsuboxyd.

Unsere weiteren Untersuchungen baben die Angaben der ersten Mittheilung in jeder Beziehung bestätigt. Wir heben noch hervor, dass grössere Mengen des Phosphorsuboxyds im Laboratorium am bequemsten vermittelst unterphosphorigsauren Ammoniums erbalten werden ${ }^{4}$ ).

\section{Phosphorsuboxyd aus Phosphor.}

Zunächst haben wir nochmals entsprechend den Angaben der ersten Abhandlung (diese Annalen 310, 56) Phosphorsuboxyd dargestellt. Nach dem Auswaschen auf Thontellern getrocknet, wurde die bräunliche, unansehnliche Masse im Achatmörser fein zerrieben. Das so erhaltene gelbrötbliche Pulver ergab nach dem Trocknen im Vacuumexsiccator neben Phosphorsäureanhydrid bis zum constanten Gewicht bei der Analyse folgende Zahlen:

$0,0990 \mathrm{~g}$ gaben $\left.0,3121 \quad \mathrm{Mg}_{8} \mathrm{P}_{2} \mathrm{O}_{7},{ }^{5}\right)$

$\begin{array}{ccc} & \text { Berechnet } & \text { (iefinde } \\ \mathrm{P} & 88,57 & 88,04\end{array}$

1) Besson (Bull. soc. chim. 1900, 582) hat neuerdings die Existen\% eines Oxydes $\mathrm{P}_{2} \mathrm{O}$ neben $\mathrm{P}_{4} \mathrm{O}$ vertheidigt. Dieses $O x y d$ bildet sich nach ihm auch durch Einwirkung von $\mathrm{PH}_{3}$ auf $\mathrm{POCl}_{3}$ und beim Einleiten ron Luft in eine Lösung von Phosphor in Tetrachlorkohlenstoff. Wir haben ein so erhaltenes $0 x_{y} d$ nicht untersucht; man sollte annehmen, dass dasselbe durch Alkali glatt und ohne Gasentwickelung in unterphosphorigsaures Salz übergefïhrt werde.

5) Zur Bestimmung des Phosphors oxydirten wir immer die Substanz mit Brom, wie in der ersten Abhaudlung seite 67 angegeben. I)urch Erhitzen der Substanz mit verdünnter Salpetersäure in zugeschmolzenen Rohre wird leicht bei directer Ausfillıng mit Magnesiumgemisch zu viel Phosphor erhalten, indem die Rohrwandung angegriffen wird. Wir fügen noch berichtigend hinzu, dass Salpetersäure von $30 \mathrm{pC}$. Gehalt auf das Phosphorsuboxyd nicht unter Feuererscheinung wirkt. 
262 Michaelis und v. Arend, Ueber das Phosphorsuboxyd.

Es war also reines Phosphorsuboxyd gebildet. Wir stellten dasselbe nun unter Bedingungen dar, die möglichst ungünstig für die Reingewinnung waren. $\mathrm{Zu}$ diesem $\mathrm{Zwecke}$ wurde die in gewöbnicher Weise unter Anwendung von granulirtem Phosphor erhaltene rothe Lösung (welche durch Papier nur langsam filtrirt) durch ein Stiick Leinwand, das so dicht war, dass Ktigelchen des gewöhnlichen Phosphors nicht hindurchgehen konnten, in ein grosses Becherglas colirt, welches überschüssige verdunnte Salzsäure, die nicht geküblt wurde, enthielt. Bei dem raschen Einlauf der alkalischen Flüssigkeit trat fühlbare Erwärmung ein, namentlich beim Umrähren. Der grünliche Niederschlag, der zwar voluminös ist, sich aber doch ziemlich leicht absetzt, wurde zuerst durch Decantiren, dann auf dem Filter mit Wasser, Alkohol, Aether nach einander gewaschen und auf Thontellern, zuletzt im Exsiccator neben Schwefelsäure getrocknet. Es ist dabei zweckmässig, die Masse auf dem Thonteller anfangs mit einem Hornspatel ofter zu verarbeiten, um Zusammenballen zu grösseren festen Stücken zu verhindern ${ }^{6}$ ), Die trockne Masse wurde im Achatmörser fein zerrieben und dann nochmals ausgewaschen, da das Trocknen grösserer Mengen des Niederschlages nur langsam erfolgt, und während dessen durch Oxydation Säuren des Phosphors (namentlich bei Sommertemperatur) entstehen können. Da das Oxyd jetzt dicht ist, erfolgt das Auswaschen (mit Wasser, Alkohol, Aether) und Trocknen viel schneller. Es wurden so vier Präparate, in derselben Weise aber getrennt, dargestellt. Zur Analyse wurde stets nur Substanz, die im luftverdünnten Raume neben Phosphorpentoxyd bis zur Gewichtsconstanz getrocknet war, angewandt.

I. $0,1016 \mathrm{~g}$ gaben $0,3316 \quad \mathrm{Ngg}_{2} \mathrm{P}_{\mathrm{q}} \mathrm{O}_{7}$.

II. $0,1142 \mathrm{~g} \quad, \quad 0,3700 \mathrm{Mg}_{8} \mathrm{P}_{\mathrm{g}} \mathrm{O}_{7}$.

III. $0,1131 \mathrm{~g} \quad " \quad 0,3710 \mathrm{Mg}_{9} \mathrm{P}_{2} \mathrm{O}_{7}$.

IV. $0,1000 \mathrm{~g} \quad, \quad 0,3279 \mathrm{Mgg}_{8} \mathrm{P}_{2} \mathrm{O}_{7}$.

6) Beim Arbeiten in Sommer ist diese Methode zweckmz̈rsiger als die des Gefrierenlassens (Seite 57 der ersten Abhandlung). 
Michaelis und v. Arend, Ueber das Phosphorsuboxyd. 263

\begin{tabular}{|c|c|c|c|c|}
\hline \multirow[t]{2}{*}{ Berechnet } & \multicolumn{4}{|c|}{ Gefinden } \\
\hline & I. & II. & HI. & IV. \\
\hline 88,57 & 91,15 & 90,48 & 91,61 & 91,57 \\
\hline
\end{tabular}

Durch eipfaches Coliren der rothen Lösung und Fällung mit yerdinnter Salzsāure, obne Küublung, war also ein Product erhalten, das $2-3 \mathrm{pC}$. Phosphor zu viel enthielt. Man kanu ein solches Präparat aber sehs leicht reinigen, indem man die in der ersten Abhandlung wiederbolt hervorgehobene Methode benutzt: nochmaliges Lösen und Fällen. Um uns zu überzeugen, dass bei dieser Operation keine selur grossen Verluste entsteben, führten wir folgenden Versuch rait analysenteinem Phosphorsuboxyd aus, das vermittelst unterphosphorigsaurem Ammonium (siehe weiter unten) erhalten war. $3 \mathrm{~g}$ desselben wurden in $100 \mathrm{ccm}$ wässrig-alkoholischen Alkalis, das vorher auf $0^{0}$ abgekühlt war, unter EisküLlung gelöst und die klare Lösung in ebenfalls auf $0^{\circ}$ abgekühlte und mit Eisstücken versetzte funfprocentige Essigsäure filtrirt. Der entstandene dunkelgrüne Niederschlag wurde, wie oben angegeben, ausgewaschen und getrocknet. Das Gewicht desselben ergab sich alsdann zu $2,5 \mathrm{~g}$, Bo dass also $83,3 \mathrm{pC}$. der angewandten Substouz wiedererbalten waren. Controlanalysen ergaben die Reimheit des Productes.

I. $0,1224 \mathrm{~g}$ gabeu $0,3872 \mathrm{Mg}_{\mathrm{g}} \mathrm{P}_{\mathbf{2}} \mathrm{O}_{7}$.

II. $0,1438 \mathrm{~g} \quad, \quad 0,4560 \mathrm{M}_{\mathrm{g}_{2}} \mathrm{P}_{2} \mathrm{O}_{7}$.

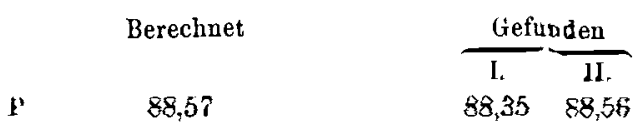

Es wurden nun die oben angeführten Präparate, die eineu zu bohen Gebalt an Phosphor ergeben hatten, derselben Reinigung unterworfen, wobei besouders dafür gesorgt wurde, dass die rothe Lösung ganz klar filtrirte, was jetzt leitbt uod zjemlich rascb von statten ging. Nach völligem Auswaschen (zweimal) und Trocknen ergaben die so gereinigten Prüparate folgende Zahlen (II war zu anderen Versuchen verbraucht). 


$$
\begin{aligned}
& \text { I. } 0,1605 \mathrm{~g} \text { gaben } 0,5043 \mathrm{Mg}_{2} \mathrm{P}_{2} \mathrm{O}_{7} \text {. } \\
& \text { III. } 0,1106 \mathrm{~g} \quad, \quad 0,3504 \mathbf{M g}: \mathbf{P}_{\mathbf{q}} \mathrm{O}_{7} \text {. } \\
& \mathrm{IV}_{\mathrm{x}} .0,1337 \mathrm{~g} \quad \text { " } 0,4235 \mathrm{Mg}_{8} \mathrm{P}_{8} \mathrm{O}_{7} \text {. } \\
& \mathrm{IV}_{\mathbf{2}} .0,1128 \mathrm{~g} \quad \text { : } \quad 0,3570 \mathrm{Mg}: \mathrm{P}_{\mathbf{2}} \mathrm{O}_{2} \text {. }
\end{aligned}
$$

Berechnet

\begin{tabular}{cccc}
\multicolumn{4}{c}{ Gefunden } \\
I. & III. & IV. & IV. \\
87,75 & 88,48 & 88,47 & 88,39
\end{tabular}

Es lag also in allen drei Fällen reines Suboxyd vor. Wenn daher zufällig nicht ganz reine Nicderschłäge erhalten werden, wie dieg anfangs von $P$ its $\mathrm{ch}$ und dem Einen von uns zuweilen und auch von $\mathrm{Cbapman}$ und Lidbury beobachtet wurde, so hat man nur nöthig, das Präparat nochmals $z u$ lösen und zu fällen, was, wie wir gezeigt haben, obne wesentlichen Verlust geschehen kann. Nothwendige Bedingung hierbei ist immer genügende Abkthblung der Lösung, da sich dieselbe bei gewöhnlicher Temperatur unter Gasentwickelung bald entfärbt.

Wodurch der zu bohe Phosphorgehalt bei der oben angegebenen Darstellung bedingt wird, ist nicht sicher anzugeben. Vielleicht rübrt er zum Theil von beigemiscbtem rothem Phosphor her, der sich bei der Oxydation des regulären Phosphors durch das Alkali bilden und fein vertheilt durch die Poren des Filters bindurch gehen kann.

In der That sind die Phosphorkügelchen, nachdem sie einigemal mit dem alkoholisch-wässrigen Alkali behandelt sind, mit einer röthlichen Schicht überzogen, welche die weitere Wirkung des Alkalis hindert. Andererseits ist in der alkoholischen Flussigkeit immer Phosphorwasserstoff aufgelöst, der durch die Wirkung der Salzsäure, namentlich beim Erwärmen, Veranlassung zur Bildung von festem Phosphorwasserstoff geben kaun.

Beim Fällen der concentrirten eiskalten rothen Lösung des Suboxyds durch Säuren ist die dunkelgrüne Farbe des Niederschlages, welche erst beim Trocknen in gelblichroth ubergeht, besonders auffallend. Es ist nicht unwalurscheinlich, dass der frisch gefäilte Niederschlag, wie Le Verrier an- 

nimmt, ein Hydroxyd $\mathrm{P}_{4}(\mathrm{OH})_{2}$ darstelle, das erst beim Trocknen in das Oxyd übergehe.

\section{Phosphorsuboxyd aus unterphosphoriger Säure.}

Bei wiederholter Darstellung des Phosphorsuboxydes nach dieser Methode hat es sich als zulässig ergeben, die Menge des Essigsäureanhydrides im Verbältniss $2 u$ der unterphosphorigen Säure gegenüber der zuerst angegebenen Vorschrift erheblich zu vermindern. Man verfährt zweckmässig wie folgt:

Die käufliche (kalkhaltige) wässrige Säure wird zunächst auf dem Wasserbade eingedampft und dann mit absolutem Alkohol und viel trocknem Aether versetzt, wodurch ein voluminöser Niederschlag von Kalksalzen ausfällt. Vom Filtrate werden Alkohol und Aether wieder abdestillirt und der Rückstand auf dem Wasserbade eingedampft. Man erhält so eine ziemlich reine Säure vom spec. Gew. 1,4625. Zur Darstellung des Suboxydes wurden $30 \mathrm{~g}$ dieser Säure in $100 \mathrm{~g}$ Eisessig gelöst und hierzu allmählich $90 \mathrm{~g}$ Essigsäureanhydrid hinzugefügt. Die Reaction verläuft dann wie in der ersten $\mathrm{Ab}$ handlung Seite 59 angegeben. Die Ausbeute beträgt 6,5 g $\mathrm{P}_{\mathbf{s}} \mathrm{O}$ oder $67 \mathrm{pC}$. der theoretischen.

I. $0,0985 \mathrm{~g}$ gaben $0,3134 \mathrm{Mgg}_{\mathrm{g}} \mathrm{P}_{2} \mathrm{O}_{7}$.

II. $0,0793 \mathrm{~g} \quad, \quad 0,2511 \quad \mathrm{Mg}_{2} \mathrm{P}_{\mathrm{g}} \mathrm{O}_{7}$.

Bereclinet

$\frac{\text { Gefunden }}{\text { I. }}$

$\mathbf{P} \quad 88,57$

$88,86 \quad 88,43$

Sehr zweckmässig kann man auch zur Darstellung des Suboxydes das leicht völlig rein zu erhaltende unterphosphorigsaure Ammonium verwenden, das aus Wasser, oder leichter aus Alkohol, in weissen Nadeln wasserfrei krystallisirt, also der Formel $\mathrm{NH}_{4} \mathrm{H}_{2} \mathrm{PO}_{2}$ eutspricht.

$0,2832 \mathrm{~g}$ gaben mit Brom und Wasser oxydirt $0,3820 \mathrm{Mg}_{2} \mathrm{P}_{2} \mathrm{O}_{2}$.

Berechnet

Y

37,35
Gefunden

37,67

Zur Darstellung des Salzes versetzt man die Lösung der unterphosphorigen Säure (oder auch die des Ca- oder Ba-Salzes) 
mit kohlensaurem Ammonium in geringem Ueberschuss, filtrirt, verdampft auf dem Wasserbade zur Trockne und bringt das abgepresste Salz in den Exsiccator. Zur Gewinnung des Phosphorsuboxydes stellten wir zwei Versuche an.

I. $30 \mathrm{~g}$ des Ammoniumsalzes wurden in $50 \mathrm{~g}$ Eisessig unter gelindem Erwärmen gelöst und $90 \mathrm{~g}$ Essigsäureanbydrid allmäblich hinzugefügt. Zuerst setzt man zweckmässig nur etwa die Hälfte des Anbydrides zu und erwärmt auf dem Wasserbade, bis die Flüssigkeit sich trubt. Dann küblt man sofort $a b$, damit die Reaction picht zu heftig wird, versetzt mit dem Rest des Anhydrides und erwärmt wieder auf dem Wasserbade. Zuletzt giesst man alles in viel Wasser, filtrirt und trocknet. Ausbeute $6, \overline{\mathrm{o}} \mathrm{g}$.

II. $30 \mathrm{~g}$ des Ammoniumsalzes wurden in $100 \mathrm{~g}$ Eisessig gelöst und dann genau so verfabren, wie unter I angegeben. Die Reaction verlief etwas heftiger. Ausbeute $9,4 \mathrm{~g}$ oder 91 pC. der theoretischen, unter der Annahme, dass die Reaction nach der Gleichung verläuft.

$$
5 \mathrm{NH}_{4} \mathrm{H}_{2} \mathrm{PO}_{2}=\mathrm{P}_{4} \mathrm{O}+\mathrm{H}_{8} \mathrm{PO}_{3}+6 \mathrm{H}_{8} \mathrm{O}+5 \mathrm{NH}_{3}
$$

Man gewinnt also nach dieser Methode am bequemsten das Suboxyd; es ist leicht, nach derselben mehrere hundert Gramm in einigen Tagen darzustellen.

Die Reinheit der Verbindung ergiebt sich aus folgender Analyse.

$0,1432 \mathrm{~g}$ gaben $0,4527 \mathrm{Mg}_{2} \mathrm{P}_{9} \mathrm{O}_{5}$.

$\begin{array}{ccc} & \text { Berechnet } & \text { Gefunden } \\ \mathrm{P} & 88,57 & 88,29\end{array}$

Das so erbaltene Suboxyd ist viel voluminoser, als das durch Fällung der rothen Lösung erhaltene und bat auch eine viel hellere Farbe. Dass beide Körper sich aber nur durch den Grad der Vertheilung unterscheiden und nicht etwa zwei physikalisch verschiedene Modificationen darstellen, ergab die Bestimmung des specifischen Gewichtes des gelben Suboxydes. Diese Bestimmung musste sebr vorsichtig ausgefuhrt werded, 
da dies Suboxyd viel Luft einschliesst und somit das specifache Gewicht leicht zu niedrig gefunden wird. Nachdem die Luft der mit Wasser übergossenen Substanz durch wiederboltes Erwärmen auf dem Wasserbade und im luftverdünnten Raume völlig entzogen war, ergab sicb das specitiscbe Gewicht zu 1,9130, beroged auf $4^{0}$. Das specifische Gawicht des ans der rothen Lösung gefällten Suboxydes wurde fruber bei $26^{\circ}$ 14 1,9123 gefunden. Erneute Bestimmungen ergaben dasselbe bei $4^{0} \mathrm{zu} 1,9116$, so dass also die nach beiden Methoden dargestellten und ausserlich verschjedenen Suboryde dasselbe specifische Gewicht besitzen.

An der Luft oxydirt sich das Suboxyd allmählich, indem Säuren des Phosphors, hauptsächlich unterphosphorige Säure entstehen, die der Substanz anbängen, obme dass dieselbe wesentlich feucht erscheint. Man kann diese Säuren durch Auswaschen ieicat entfernea. Wir haber 2. B. ein Präparat untersucht, das etwa ein halbes Jahr lang in einem Glasrohre, das nur lose mit einem Korkstopfen verschlossen war, auf. bewahrt wurde.

Beim Auswaschen gabcs $0,7750 \mathrm{~g}$ ein Filtrat, das nacb der Oxydation mit Brom 0,2359 g $\mathrm{Mg}_{2} \mathrm{P}_{3} \mathrm{O}_{7}$ lieferte, entsprecbend 8,51 pC. P. Bezogen auf unterphosphorige Saure würde dieses Präparat also $18,1 \mathrm{pC}$. der genannten Säure enthalten haben. Nach dem Trocknen gab die ausgewaschene Substanz bei nochmaligem Auswaschen ein Filtrat, das nur noch $0,0081 \mathrm{~g} \mathrm{Mg}_{8} \mathrm{P}_{2} \mathrm{O}_{7}$ lieferte, entsprecbend 0,29 pC. $P$ oder 0,62 pC. unterphosphorige Säure. Die Aoalyse des Suboxydes ergab:

$0,1127 \mathrm{~g}$ Gaben $0,3562 \mathrm{Mgg}_{\mathrm{g}} \mathrm{P}_{\mathrm{g}} \mathrm{O}_{7}$.

$\begin{array}{cc}\text { Berechnet } & \text { Gefundeu } \\ 88,57 & 88,27\end{array}$

Der gesioge Gehalt an unterphosphoriger Säure drbckt sich in der zu niedrig gefundenen Menge des Ptosphors aus ${ }^{7}$ ).

7) Zieht man ron der Substanzmenge $0,62 \mathrm{pC}$. und von der $0,3562 \mathrm{~g}$ $\mathrm{Mg}_{2} \mathrm{P}_{9} \mathrm{O}_{7}$ entsprechenden $\lambda$ renge Phosphor $(0,0994792 \mathrm{~g}) 0,29 \mathrm{pc}$. $a b$, so ergiebt sich ein I'rncentgehalt von 88,52 . 
Hieraus geht hervor, dass man anbängende Säuren des Phosphors bis auf geringe Mengen (die sich beim Trockuen wieder bilden) leicht entfernen kann. Es ist daher die Annabme von Chapman und Lidbury, die Differenz von etwa 10 pC. Phosphor, die von ihnen bei der Analyse des aus der rothen Lösung gefällten Körpers gegenüber amorphem Phosphor erhalten wurde, sei durch einen Gehalt an Säuren des Phosphors veranlasst, durchaus hinfällig.

Das trockne Phosphorsuboxyd kann in einer Koblensäureatmosphäre läugere Zeit auf die Temperatur des siedenden Wassers erhitzt werden, ohne sich wesentlich zu verändern. Wurde es in dieser Weise 12 Stunden erhitzt, so enthielt das Filtrat beim Auswaschen 0,47 pC. Phosphor in Form von Säuren des Phosphors. Erbitzt man das Suboxyd mit Wasser im Rohre in einer Kohlensäureatmosphäre, so erfolgt die Oxydation auch nur langsam.

$0,5247 \mathrm{~g}, 12$ Stunden in der angegebenen Weise erhitzt, lieferten ein Filtrat, das nach der Oxydation mit Brom $0,0318 \mathrm{~g} \mathrm{Mg} \mathrm{Mg}_{8} \mathrm{O}_{7}$ ergab oder 1,69 pC. Phosphor. Der Rïckstand enthielt $89,04 \mathrm{pC}$. Phosphor, also etwas mehr als die berechnete Zahl $(88,57)$.

Auch bei dem Auswaschen des Suboxydes mit kochendem Wasser und kochendem Alkobol wird es nicht verändert. Der Phosphorgehalt eines so behandelten Suboxydes ergab sich zu 88,9:, war also nur wenig höher als der berechnete.

Zum Schluss heben wir noch hervor, dass das Phosphorsuboxyd (in der einen oder anderen Weise dargestellt) sich in einer eiskalten Lösung von alkoholisch-wässrigem Alkali immer olne Gasentwickelung und so schnell etwa löst, wie zerriebenes Kochsalz in Wasser, im Gegensatz zu dem Phosphor selbst, der sich immer mit Gusentwickelung und nur sehr langsam (nie vollständig, da er sich immer mit einer rothen Schicht überzieht) löst. Diese Thatsache erscheint uns von Wichtigkeit, da, wenn der rothe Phosphor in irgend einer Form (bis jetzt ist keine solcbe festgestellt) in alkoholisch-wässrigem Alkali löslich sein sollte, diese Auflösung sicherlich ebenfalls wie die des regulären Phosphors unter Gasentwickelung erfolgen würde. 
Michaelis und v. Arend, Ueber das Phosphorsuboxyd. 269

Zur sicheren F'eststellung dieses Verhaltens benutzten wir den in Fig. 1 abgebildeten Apparat. In den Kolben $A$ wurden etwa $50 \mathrm{ccm}$ Alkohol gebracht, das Ganze auf $0^{\circ}$ abgekühlt, alsdann das Phosphorsuboxyd hinzugebracht und dies durch Schütteln möglichst vertheilt. In den Hahntrichter B brachten wir eine auf $0^{\circ}$ abgekühlte (und mit einigen Eisstückchen versetzte) Mischung von einem Volum Alkohol und einem Volum

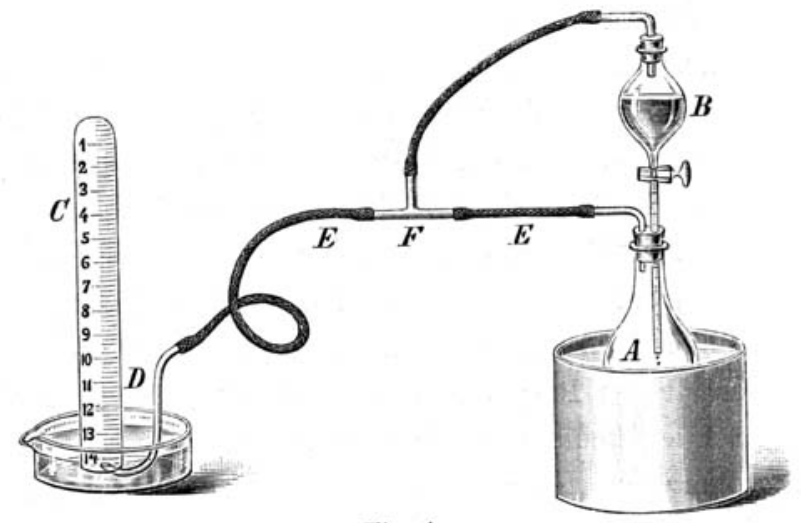

Fig. 1.

Natronlauge und schoben das getheilte und mit Wasser gefullte Rohr C über die Mündung der Gasleitungsröbre $D$; welche durch den Schlauch EE mit dem Kolben A verbunden war. Dieser Schlauch stand durch das T-Rohr F auch mit dem Hahntrichter in Verbindung. Wurde nun der Hahn des letzteren geöffnet, so gelangte die alkalische Flüssigkeit in den Kolben $A$ obne Aenderung des Gesammtvolumens. Die Lösung erfolgte nun bei gelindem Umscbütteln (durch Anfassen an dem vorstehenden Rand des Kolbens) rasch und ohne dass mehr als 0,5 bis $1 \mathrm{ccm}$ Gas in das getheilte Rohr eintrat, eine Volumänderung, die auch durch die geringste Erwärmung hervorgebracht wird. Frst beim Stehen der dunkelrothen Lösung begann eine sehr langsame Gasentwickelung, die sich beim Herausnehmen des Kolbens $A$ aus dem Eiswasser rasch verstärkte, indem allmählich Entfärbung der Lösung eintrat. 
3. Versuche zur Darstellung reinen amorphen Phospliors durch Belichtung des regulären Phosphors.

Wie schon in dem Eingange dieser Abhandlung angeführt wurde, stellte zuerst Alexander $\mathrm{Pedler}^{8}$ ) grössere Mengen rothen Phosphors durch die Wirkung des Lichtes dar. Er exponirte entweder concentrirte Lösungen vou Phosphor in Schwefelkohlenstoff (theils für sich, theils unter Zusatz von etwas Jod oder Phosphortribromid) oder Lösungen des Phosphors in Phospbortrichlorid, Benzol und Chloroform dem Sonnenlichte oder er setate direct den festen Phosphor der Wirkung des Lichtes im Vacuum der Sprengel'schen Quecksilberluftpumpe aus. In fast allen Fällen erhielt er ein unter dem Mikroskop krystallinisch erscheinendes Pulver, das aus einem Gemisch von rothen und gelben Partikelchen bestand. Nur der aus Chloroform abgeschiedene Phosphor erscbien amorph. Alle so erhaltenen Präparate waren ganz untöstict in Aikoboi, Aether, Schwefelkohlenstoff, dagegen löslich unter Entwickelung von Phosphorwasserstoff in warmer Sodalösung. Eine Bestimmung des specifischen Gewichtes oder eine qualitative und quantitative Analyse wurde nicht ausgefübrt.

Wir baben bis jetzt im Wesentlichen nur mit Lösungen des Phosphors gearbeitet. Während Pedle $r$ durch die Wirkung der tropischen Sonne augenscheinlich eine ziemlich gute Ausbeute an rothem Phosphor erbalten hat, war die Menge, die wir durch die bei weitem weniger wirksamen Sonnenstrahlen unserer Breitengrade erbielten, immer nur sehr gering.

Um den Grad der Reinheit von rothem Phosphor uberhaupt kennen zu lernen, bestimmten wir den Phosphorgehalt verschiedener käuflicher Präparate, die zur Analyse ausgewaschen und getrocknet wurden. Von der Firma Schuchardt, Görlitz, bezogener rotber Pbosphor entbielt 98,74, von Kablbaum, Berlin, bezogener Phosphor 99,56 und ein im hiesigen chemischen Institut schon seit längerer Zeit vorhandenes Präparat

9) Journ. chem. Soc. Transact. 57, 599. 
unbekannter Herkunft $96,94 \mathrm{pC}$. Phosphor. Der Arsengehalt (durch tagelanges Einleiten von Schwefelwasserstoff in die mit Salzsäure und chlorsaurem Kalium erhaltene und durch Erhitzen von freiem Chlor völlig befreite warme Lösung bestimmt) war in allen Fälled nur gering. Wir versuchten nun einen rothen Ptosphor von äbnlicher Reinheit durch die Wirkung des Lichtes $z u$ erhalten.

I. Versuch. 100 Gewichtstheile regulären Phosphors, der mit Filtrirpapier getrocknet war, wurden in 100 Theilen reinem, mit Chlorcalcium getrocknetem Schwefelkohlenstoff gelöst, die Lösung filtrirt una in einem Stöpselglase, das bis auf eine bleine Luftblase ganz mit der Lösung gefüllt war, drei Wochen lang dem Sonnenlichte ausgesetzt. Es hatte sich dann theils an der Wand, theils an dem Boden des Gefässes ein rother Niederschlag abgesetzt, der zuerst mit Schwefelkohlenstoff, dann mit Alkohol, darauf mit Wasser und zuletzt wieder mit Alkobol gewaschen und im Vacuumexsiccator neben Phosphorpentoryd getrocknet wurde. Die qualitative Analyse ergab die Anwesenheit von Schwefel, auch roch die Substanz an feuchter Luft deutlich nach Schwefelwasserstoff. Es ist dies nicht zu verrundern, da es bekannt ist, dass Schwefelkoblenstoff durch das Licht verändert wird, indem sich freier Schwefel bildet ${ }^{9}$ ). Die quantitative Analyse fuhrte zu folgenden Zahlen:

I. $0,1065 \mathrm{~g}$ gaben $0,3106 \mathrm{Mg}_{2} \mathrm{P}_{\mathrm{2}} \mathrm{O}_{7}$.

II. $0,0942 \mathrm{~g} \quad, \quad 0,2757 \mathrm{Mg}_{2} \mathrm{P}_{2} \mathrm{O}_{7}$.

III. $0,1169 \mathrm{~g}, 0,0690 \mathrm{BaSO}$.

IF. $0.1962 \mathrm{~g}: 0,1104 \mathrm{BaSO}_{4}$.

\begin{tabular}{cccccc} 
& \multicolumn{5}{c}{ Gefunden } \\
\cline { 2 - 6 } I. & I. & II. & III. & IV. & Mittel \\
s. & 81,45 & 81,66 & & - & 81,55 \\
S & - & $\ldots$ & 8,11 & 7,73 & 7,92
\end{tabular}

Das Präparat musste danach auch sauerstoffhaltig sein, da die Gesammtsumme der vorhandenen Elemente im Mittel nur 89,47 betrug, also noch eine Differenz von $10,53 \mathrm{pC}$.

9) Vergl. 2. B. Sidot, Compt. rend. 69,$1303 ; \mathbf{7 4}, 180 ; 81,32$. 
gegen 100 vorhanden war, die nur von aufgenommenem Sauerstoff herrühren konnte. In der That war die Substanz auch mit rother Farbe in wässrig-alkoholischem Alkali löslich und auf Zusatz von Säuren wurde aus dieser Lösung ein grünlicher Niederschlag gefällt. Woher stammte aber dieser Sauerstoff, da die atmosphärische Luft doch so gut wie abgeschlossen war? Während der Belichtung der Lösung hatten wir bereits die Beobachtung gemacht, dass in dem Glase (auch wenn es durch Einstellen in Wasser gekühlt wurde) Druck vorhanden war und beim Oeffuen trat deutlich der Geruch nach Phosphorwasserstoff auf. Dieses Gas konnte nur unter Mitwirkung von Feuchtigkeit entstanden sein und von dieser auch der Sauerstoffgehalt der Verbindung herrühren. Diesem Fingerzeige entsprechend stellten wir folgende Versuche an:

II. Fersuch. Eine 50 procentige Lösung von regulärem Phosphor in reinem Schwefelkoblenstoff wurde mit Phosphorpentoxyd getrocknet, filtrirt und dann ein Einschmelzrohr etwa bis zu Zweidrittel mit dieser Lösung gefüllt ${ }^{10}$ ). Die in dem Robre enthaltene Luft wurde durch Einleiten von trocknem, durch glühendes Kupfer völlig luftfrei erbaltenem Stickgas verdrängt, das Rohr dann zugeschmolzen und 14 Tage dem directen Sonnenlichte ausgesetzt, wobei sich nur sehr langsam ein rothgelber Anflug an der Rohrwand bildete, der allmählich abblätterte. Beim Oeffnen zeigte das Rohr keinen Druck. Der feste rothe Körper enthielt, nachdem er, wie unter I angegeben, gewaschen und getrocknet war, der qualitativen Analyse zufolge neben Phosphor Schwefel und Kohlenstoff. Die quantitativen Bestimmungen konnten bei der geringen Menge des vorhandenen Materials nur mit sehr kleinen Substanzmengen ausgeführt werden.

I. $0,0838 \mathrm{~g}$ gaben $0,2226 \mathrm{Mg}_{8} \mathrm{P}_{9} \mathrm{O}_{3}$.

II. $0,0458 \mathrm{~g} \quad, \quad 0,1211 \mathrm{Ng}_{2} \mathrm{P}_{2} \mathrm{O}_{7}$.

III. $0,0916 \mathrm{~g}, 0,0930$ BaSO $_{\text {. }}$.

IV. $0,0789 \mathrm{~g},, 0,0205 \mathrm{CO}_{\mathrm{g}}$.

10) Man muss sehr vorsichtig mit einer solchen Lösung arbeiten, da sie sich leicht entzündet. 


\begin{tabular}{ccccc} 
& \multicolumn{5}{c}{ Gefunden } & \\
\cline { 2 - 5 } P & I. & II. & III. & IV. \\
s & 74,19 & 73,85 & - & - \\
r: & $\ldots$ & $\ldots$ & 13,94 & - \\
& $\ldots$ & $\ldots$ & - & 7,09
\end{tabular}

Die Gesammtsumme der quantitativ bestimmten Elemente beträgt 94,07 (für $\mathrm{P}$ als Mittel 74,04 pC. gerechnet), so dass also auch noch etwas Sauerstoff in dem Präparate enthalten sein konnte, der möglicher Weise beim Waschen mit Wasser durch Ersatz ron Schwefel aufgenommen war.

Wurde etwas dieses Präparates im Reagensglase mit wässrigalkoholischem Alkali übergossen, so trat keine Rothfärbung ein, sondern dasselbe ging farblos unter Gasentwickelung in Lösung. Wenn das Präparat jedoch im Achatmörser fein zerrieben wurde, so erfolgte mit dem angegebenen Lösungsmittel schwache Rothfärbung. Wurden gleiche Gewichte dieses Präparates und reinen Phosphorsuboxydes (jedes für sich) mit der alkalischen Flüssigkeit übergossen, so war der Unterschied in der Rothfärbung sehr gross.

III. Versuch. Es wurde in ein Einschmelzrohr in derselben Weise wie bei II eine trockne Phosphorlösung gebracht, aber die Luft nicht durch Stickgas verdrängt. Der nach 14 tägiger Belichtung erhaltene rothe Phosphor zeigte ganz ähnliche Eigenschaften wie der unter II beschriebene.

$0,1092 \mathrm{~g}$ gaben $0,0896 \mathrm{BaSO}_{4}$ und $0,3006 \mathrm{Mg}_{2} \mathrm{P}_{8} \mathrm{O}_{7} \cdot{ }^{11}$ )

$\begin{array}{cc} & \text { Gefunden } \\ \text { l } & 76,89 \\ \mathrm{~s} & 11,27\end{array}$

Der Kohlenstoff konnte aus Mangel an Material nicht bestimmt werden.

IV. Versuch. Es wurde ejn Einschmelzrohr bis zu Zweidrittel mit einer klaren 50 procentigen Lösung von Phosphor in Schwefelkohlenstoff gefullt, etwas Wasser hinzugefügt und

12) Bei der geringen Menge Substanz, die erhalten wurde, mussten beide Elemente in derselben Substanzmenge bestimmt werden. 
274 Michaelis und v. Arend, Ueber das Phosphorsuboxyd.

die Luft durch Stickgas verdrängt. Bei der Belíchtung, die ebenfalls 14 Tage währte, bildete sich viel schueller als bei den Versuchen II und III ein zuerst rein gelber, allmählich orangeroth werdender, ziemlich voluminöser Niederschlag. Beim Oeffnen zeigte das Rohr deutlich Druck.

$0,1081 \mathrm{~g}$ gaben $0,2980 \mathrm{M}_{\mathrm{S}_{\mathbf{4}}} \mathrm{P}_{\mathbf{2}} \mathrm{O}_{7}$ und $0,0930 \mathrm{BaSO}_{4}$.

$$
\begin{array}{lc} 
& \text { Gefunden } \\
\mathrm{P} & 76,99 \\
\mathrm{~S} & 11,82
\end{array}
$$

Dieses Präparat löste sich in wässrig-alkobolischem Alkali leicht mit intensiv rother Farbe.

V. Versuch, Eine Stange gewöhnlichen Phosphors wurde unter Wasser drei Wochen lang dem directen Sonnenlichte ausgesetzt und der gebildete rothe Ueberzug durch Behandeln der Phosphorstange mit reinem Scbwefelkohlenstoff und Auswaschen des Rückstandes mit diesem, Alkohol u. s. w. isolirt. Der schwefelfreie Körper zeigte fast genau die Zusammensetzung deg Phosphorsuboxydes.

$0,0855 \mathrm{~g}$ gaben $0,2722 \mathrm{Mg}_{8} \mathrm{P}_{\mathbf{8}} \mathrm{O}_{7}$.

$\begin{array}{ccc} & \text { Berechnet } & \text { Gefunden } \\ l^{\prime} & 88,57 & 88,91\end{array}$

Er löste sich klar und ohne Rückstand in wässrig-alkobolischem Alkali.

Durch diese Versuche ist erwiesen, dass der Phosphor im Sonnenlichte das Wasser zersetzt unter Bildung von $\mathrm{P}_{4} \mathrm{O}$ :

$$
\mathrm{P}_{4}+\mathrm{H}_{2} \mathrm{O}=\mathrm{P}_{4} \mathrm{O}+\mathrm{H}_{2} \text {. }
$$

Der Wasserstoff wird dann theilweise in Phosphorwasserstoff ubergeführt.

Bei Gegenwart von Feuchtigkeit wird also aus einer Phosphorlösung immer neben rothem Phosphor auch Phosphorsuboxyd gebildet, so dass das Präparat alkoholisch-wässriges Aikali roth fărben muss.

VI. Versuch. In ein Einschmelzrohr wurde mit Phosphorsäureanhydrid getrockneter Tetrachlorkoblenstoff gebracht und einige Phosphorstücke, die mit Filtrirpapier getrocknet und 
Michaelis und v. Arend, Ueber das Phosphorsuboxyd. 275 dann mit trocknem Aether und trocknem Tetrachlorkohlenstoff abgespült waren, hinzugefugt. Die Luft, die in dem Rohre entbalten war, verdrängten wir durch trocknen reinen Stickstoff und exponirten das zugeschmolzene Rohr drei Wochen lang dem Sonnenlichte. Es wurden so etwa $6 \mathrm{cg}$ eines rothen Körpers erhalten, der auch kein reiner rother Phosphor war, sondern neben diesem Kohlenstoff enthielt. Bei der Oxydation desselben mit Brom und Wasser ging zuerst alles in Lösung, beim Verdunsten des Broms schied sich aber ein weisser, voluminöser Körper aus, der Kohlenstoff, Halogen und wahrscheinlich auch Phosphor enthielt.

$0,0485 \mathrm{~g}$ gaben $0,1070 \mathrm{Mg}_{2} \mathrm{P}_{\mathrm{g}} \mathrm{O}_{7}$.

$$
\text { P } \quad 61,62
$$

Der Körper färbte alkoholisch-wässriges Alkali, auch wenn er fein zerrieben wurde, keine Spur roth.

Diese Versuche ergeben, dass es keineswegs leicht ist, nahezu analysenreinen rothen Phosphor durch Belichtung, wenigstens in unseren Breitengraden zu erbalten. Möglicherweise überwiegt unter der Wirkung der tropischen Sonne bei Belichtung einer Lösung von Phosphor in Schwefelkohienstoff die Bildung des rothen Phosphors die Zersetzung des Schwefelkohlenstoffes, so dass ein reineres Product erhalten wird, als wir es zu gewinnen vermochten. Wir werden im nächsten Sommersemester versuchen, durch Belichtung ganz trocknen, regulären Phosphors (ganz ohne Lösungsmittel) in einer luftfreien Atmosphäre reinen rothen Phosphor zu erhalten. 